Voix et Images

voixetimages

\title{
La meule et la rivière. Souvenirs de Saint-Eustache
}

\section{André Brochu}

Volume 5, numéro 3, printemps 1980

\section{Fernand Ouellette}

URI : https://id.erudit.org/iderudit/200234ar

DOI : https://doi.org/10.7202/200234ar

Aller au sommaire du numéro

\section{Éditeur(s)}

Les Presses de l'Université du Québec

ISSN

0318-9201 (imprimé)

1705-933X (numérique)

Découvrir la revue

Citer cet article

Brochu, A. (1980). La meule et la rivière. Souvenirs de Saint-Eustache. Voix et Images, 5(3), 569-582. https://doi.org/10.7202/200234ar d'utilisation que vous pouvez consulter en ligne.

https://apropos.erudit.org/fr/usagers/politique-dutilisation/ 


\section{APARTE}

\section{La meule et la rivière Souvenirs de Saint-Eustache}

"Elle fut magnanime, héroïque et sans tache, / Votre légende, ô fiers enfants de Saint-Eustache! "s'exclame Louis Fréchette au début d'un poème où "morne" rime avec "Colborne", et "anglaise" avec "fournaise". SaintEustache est tout entier dans ces deux vers, épique et bon enfant, fier surtout que l'Histoire-avec-majuscule ait consigné son nom dans ses registres. Avec sa poignée de patriotes, Chénier rééditait l'exploit de Dollard des Ormeaux, par son sacrifice il sauvait la colonie qui bénéficierait sous peu de libertés politiques nouvelles. Cela n'aboutit, hélas, qu'à la Confédération, après quoi Chénier devait rester un symbole, comme Louis Riel, c'est-à-dire un exemple ambigu, utilisé à toutes les sauces. A Saint-Eustache il a sa rue, sa "place" qui, bien entendu, est plutôt un édifice commercial, sa statue devant l'église. Libéraux, unionistes, péquistes lui rendent des hommages différents, et personne ne songe à rapatrier la fameuse urne qui contiendrait ses cendres. Cela, sans doute, sent trop son hérétique, même après que l'incinération soit devenue le dernier cri en matière d'autodétermination mortuaire.

Cher Saint-Eustache! Non seulement tu as eu tes héros, non seulement tu as été chanté par le plus éloquent de nos poètes nationaux, mais tu as toimême eu tes poètes. Ils publiaient régulièrement dans la Victoire, organe local de l'Union nationale. L'un d'eux était épicier, ce qui n'est pas sans intérêt: Crémazie, en effet, s'était plaint à l'abbé Casgrain du malheur d'être écrivain, dans une société d'épiciers. Or qu'arrive-t-il quand ces honnêtes marchands, s'élevant au-dessus des denrées périssables, se mettent à nous servir les nourritures spirituelles? II se produit exactement ceci :

O temps, suspends ton vol! heures propices, Laissez-nous savourer les rapides délices Du plus beau temps de nos jours!

Lamartine, certes, avait fait mieux, mais en moins original!

Je me souviens aussi de l'immense panneau-réclame qui faisait la publicité de la piscine municipale. C'est à Paul Valéry, auteur d'un "Cimetière marin » dont on méconnaît généralement les beautés pratiques, que les édiles avaient emprunté le vers suivant: "Courons à l'onde en rejaillir vivant !" Bien 
entendu, le pluriel de majesté était devenu un pluriel tout court, et on avait ajouté un «s " à « vivant ". Autrement, on eût risqué de jeter quelque confusion dans la compétence grammaticale des baigneurs.

Il n'y a rien d'étonnant que, étant né dans une ville si favorable aux lettres, j'ai moi-même succombé à l'appel de la Muse. C'est sans doute l'esprit de la Rébellion qui me fera terminer un de mes poèmes par un "Vive la Révolution!» digne de Fréchette lui-même, sinon de Chénier... Pourtant, lorsque je me recueille et feuillette l'album de mes souvenirs, ce sont surtout des images de paix qui me reviennent, en association avec la rivière, large comme trois fleuves français, qui coulait paresseusement derrière chez nous. C'est la rivière des Mille-îles, qui naît environ deux kilomètres en amont, alimentée par le lac des Deux-Montagnes dont elle est séparée par des rapides. Cette rivière est comme la basse continue de mon enfance, tantôt grise quand le vent d'est nous apportait de la pluie pendant trois jours, tantôt frémissante de soleil, capricieuse comme toutes les rivières et toutes les enfances. C'est elle que je voudrais évoquer d'abord, car elle est ma véritable patrie. D'aucuns ont fondé leur vie sur du sable, et d'autres sur le roc. Moi, j'ai fait fond sur l'eau, ma maison est un navire et je dérive doucement, au gré des pluies et du beau temps.

Pour la retrouver, ma rivière d'autrefois, il me faut la dépouiller de ses apparences actuelles, oublier ses eaux lourdes de détergent, les résidences cossues qui ont embourgeoisé ses rives, et surtout, il faut la replanter de joncs et de nénuphars. Cette royale végétation, qui abritait tout un peuple de grenouilles, de carouges, de pluviers, de butors et parfois, quand les dieux nous étaient favorables, de grands hérons, a complètement disparu. Disparues aussi, les demoiselles grosses comme le doigt qui piquaient sur vous avec des élégances d'aéroplanes de la première guerre, et les libellules bleues et rouges qui semblaient défendre, parmi les herbes nonchalantes, la cause héréditaire de l'Union nationale ou du parti libéral.

Quand mon père fit bâtir maison, un an avant ma naissance, le bord de l'eau n'était pas défriché. C'était un bois, comme tous les bois, rempli d'arbres grands et petits. II y en avait une assez grande variété: des ormes, des frênes, une sorte d'érables qu'on appelait des plênes et, sans doute parce que la nature aime à rimer, un ou deux chênes, un certainement qui est toujours là et que la corde à linge relie à la galerie arrière de la maison, comme un toutou tenu en laisse, depuis bientôt quarante ans.

Quand je parle du bord de l'eau, il est important de comprendre qu'une pente assez forte en sépare la portion de terrain qui entoure la maison, de sorte que nous n'avons jamais été exposés aux inondations qui, tous les printemps, désolent les environs. Il faut comprendre aussi le contraste remarquable que formait l'état des choses au printemps, où l'eau montait jusqu'à mi-côte, et au mois d'août, quand la rivière prenait presque des allures de gros ruisseau, poussant ses eaux tièdes entre ses rives de vase dont le soleil cuisait les lourds parfums. 
Le retrait des eaux, en été, dégageait une vaste étendue de terrain dont les joncs s'emparaient, jusqu'à ce que la faux les couche sur le sol. Nous, les enfants, nous en faisions alors le revêtement de nos huttes. Les jeunes arbres du sous-bois en avaient fourni les montants. Nous n'éprouvions aucun remords à abattre ces sauvageons, à l'aide de nos hachettes et de nos couteaux. L'esprit des défricheurs s'étendait jusqu'à nous. Le respect de la nature n'était nullement dans les mœurs de l'époque. D'ailleurs, chaque été, mon père et le voisin faisaient reculer un peu plus la végétation sauvage, de sorte que la pelouse a fini par rejoindre la rivière. La rançon de cette expansion de l'espace domestique fut une érosion qui donne déjà des allures de côte à ce qui, il y a trente ans, constituait, derrière la maison, un terrain tout à fait horizontal.

Blottis dans nos huttes, qui ouvraient du côté de la rivière, n'ayant plus sous les yeux l'odieux spectacle de la civilisation blanche, nous somnolions en écoutant le coassement incongru des "ouaouarons" et "patarams", et en tirant quelques bouffées de nos calumets de paix bourrés de feuilles d'érable de l'année précédente. Étourdis des odeurs de l'été, nous rêvions de chasses en pleine forêt et de massacres splendides dont nous aurions remporté quantité de scalps et autres trophées. Non, ce n'était pas l'esprit des Patriotes mais plutôt celui des Iroquois qui s'emparait de nous dans ces tranquilles après-midis de juillet que dérangeait seule, sur le coup de quatre heures, l'interminable mélopée de bronze annonçant le baptême d'un autre Blanc.

Mes rapports avec la rivière n'en furent pas de pure contemplation. On ne naît pas impunément au bord d'un cours d'eau navigable et, en ce temps-là du moins, propre à la natation, pour ne pas dire propre tout court. Très tôt, je sus manœuvrer les rames et diriger le canot, que nous appelions "chaloupe", à travers les courants. Ces courants, du reste, n'avaient pas la violence de ceux de la rivière des Prairies, qui sépare les îles de Montréal et de Laval. La rivière des Mille-îles a, dans son écoulement, quelque chose de souverain qui est dû à sa largeur, mais elle n'a rien de turbulent et, chaque hiver, une épaisse couche de glace la recouvre. C'est dire sa sérénité - sérénité de jeune géante capable, quand elle veut, de magnifiques courroux.

Au bout de la corde qui nous servait d'amarre, nous attachions, en guise d'ancre, une pierre et nous pouvions ainsi immobiliser le canot pour nous adonner au noble sport de la pêche. Nos cannes avaient la méme provenance que les montants de nos huttes, nos arcs, nos flèches et nos sagaies. Nous y fixions une ficelle avec un bouchon de liège, un hameçon et un poids de plomb; les vers provenaient du potager, dont la glaise bien grasse regorgeait. Nous enfilions le ver éperdu sur le crochet, lavions nos doigts dans la rivière et attendions, l'âme baveuse, les secousses du bouchon. Tout d'un coup ça y était, une petite frénésie s'emparait du liège, le faisait se dresser puis s'enfoncer. C'était le branle-bas dans le canot, nous devenions tout action, l'esprit collé aux profondeurs d'où nous voyions surgir l'éclair rouge et blanc d'un ventre de perchaude. C'était grand comme la main, ça faisait des sauts de carpe au fond du bateau où nous l'avions tirée - par esprit d'imitation sans doute ou pour nous consoler de l'absence de carpe dans notre rivière. Le 
menu frétin, tel était notre lot, perchaudes, barbotes, crapets-soleil. J'ai entendu parler de prises plus glorieuses, achigans ou dorés, mais la seule capture un peu exceptionnelle que nous ayons faite est celle d'une anguille. II y avait aussi, paraît-il, des barbues, au nom fort inexact puisque la barbue est un poisson de mer, et elles m'inspiraient beaucoup de respect bien que je n'en aie jamais vues. Elles évoquaient pour moi l'idée de barbotes mais en plus gros et en plus sévère, l'idée de barbotes grand-mères au regard profond et à la mastication grave.

J'ai appris à préparer les perchaudes pour la cuisson. Quand, mes frères et moi, nous en avions pêché trois au quatre, je les vidais, les écorchais, coupais têtes, queues et nageoires et apportais ce qui restait, quelques grammes de chair blême, à ma mère qui, obligeamment, les faisait cuire. Il en résultait deux ou trois bouchées pour chacun, que nous avalions avec le sourire de martyrs canadiens à qui on aurait fait ingurgiter des pelotes d'épingles. L'anguille, elle, a laissé des souvenirs vivaces dans mon esprit. L'apprêter avait été toute une affaire. Impossible de la dévêtir de sa peau, elle y tenait comme trois bourgeois. Il a fallu la clouer à un madrier, par une pointe enfoncée en pleine tête, et la dépiauter avec des pinces, en tirant de toutes nos forces. La queue contenait du sang qui nous éclaboussa. Dans mon assiette, je lui trouvai un de ces goûts d'huile qui vous remontent dans le nez, pareil à celui de l'huile de fole de morue qui avait empoisonné quelques années de mon existence. Il paraît que c'est un mets de choix. Encore faut-il aimer le poisson, et les repas du vendredi me l'avaient fait prendre en horreur. Je n'avais pas un estomac très catholique.

Mes rapports avec les poissons se limitent à ces quelques contacts, et abstraction faite de mon signe astrologique qui est précisément le signe des poissons, ils ne s'étendent pas jusqu'au niveau métaphorique. Par cette transition un peu lourde, n'est-ce pas, je veux introduire un autre aspect de mon commerce actif avec la rivière, à savoir la natation, et suggèrer que, bien loin de nager comme un poisson, j'avais plutôt la grâce d'un petit chien. En communauté avec le voisin, qui avait fourni les barils, nous possédions un radeau sur la rivière, sorte de plate-forme carrée à laquelle nous amarrions le canot et d'où nous pouvions plonger. Une échelle permettait de descendre au bain en douceur, un échelon nous amenait l'eau jusqu'au jarret, le suivant jusqu'aux fesses - brrrrrr! - , l'autre jusqu'au petit cœur, que l'eau glaçait d'une étreinte mortelle (!), et nous voilà lâchés, caniches grelottants, dans une onde mauvaise à boire. Après deux ou trois minutes, la torture des premiers moments avait fait place aux délices vivifiants de la baignade. Le plaisir consistait à plonger tout au fond et à en rapporter des moules d'eau douce, ou plutôt leurs écailles, qui étaient toujours vides. Était-ce bien des moules: Pas des huitres en tout cas, comme nous le croyions alors, notre compétence lexicale comportant en effet un grand nombre d'actes de foi. Marron foncé à l'extérieur, couleur d'aurore triste à l'intérieur, ces écailles semblaient nées de la vase d'où nous les avions extraites. II n'empêche, elles nous faisaient rêver. C'était comme si la mer, à travers elles, s'annonçait jusqu'à nous. Elle nous dépêchait aussi, du fond lointain du golfe, ses goélands criards. Rina Lasnier les chante dans de beaux vers: 
Du fleuve à la rivière en des multitudes d'eaux,

De l'épaule foisonnante de la mer à la pierre sans nourriture,

La mouette a cloîtré sur soi ses ailes à défricher les ciels...

Le poème se termine ainsi:

La mouette assise dort dans les limbes de la rivière;

Elle tarit sur soi la mue des eaux en courantes lumières,

Source d'émail blanc peinte sur la pierre.

Toute la poésie triste de ces oiseaux en exil dans nos eaux trop douces, par je ne sais quelle absurde vocation semblable à celle qui, naguère, menait au cloître de belles jeunes filles, est contenue dans ces quelques mots.

Je me rappelle mes efforts, toujours vains, pour les approcher en canot. Images de la transcendance la plus plate, les goélands sont bien les frères de Jonathan Livingstone, ce blanc dadais américain, champion du travelling et des extases à bon marché.

Pêcher, nager, canoter, ces occupations, vieilles comme le monde, gardaient bien assez d'attrait pour mon âme riveraine. Mais je ne dédaignais pas la modernité, laquelle s'offrit à nous sous l'espèce d'un hors-bord. C'était, alors, une véritable rage. II n'était pas de propriétaire, le long de la rivière, qui ne possédât le sien. J'appris assez tôt à le conduire. Mes frères et moi faisions du ski nautique, et nous nous relayions au volant. Le radeau nous servait de plate-forme de départ. Le bateau s'éloignait d'abord doucement puis, une fois la corde tendue, faisait un bond en avant qui arrachait le skieur à son appréhension: partira? partira pas ?... c'est parti! Le succès de l'opération tenait à la coordination entre les mouvements du bateau et ceux du skinaute, lequel se retrouvait parfois au niveau d'un scaphandrier! Je maîtrisai assez bien le ski normal, sur deux patins, mais ne passai jamais à l'autre échelon, celui du monoski, contrairement à mes frères qui étaient plus sportifs que moi. Le fin du fin, le chic du chic, c'était le ski sur la plante des pieds : un seul athlète, Guy Cloutier, le pratiquait dans la région. Je l'ai vu à l'œuvre derrière chez nous, lors de la fête nautique annuelle dont il constituait la principale attraction. Cette fête comportait la bénédiction des bateaux, et des régates. Les petits skiffs, bruyants et rapides, étaient particulièrement appréciés.

Notre embarcation était relativement modeste, mais très fiable, et le moteur, d'une puissance moyenne, tournait bien. Le démarrage était parfois laborieux. II fallait tirer une corde, d'un mouvement ferme et continu, rapide mais pas trop, en prenant garde de ne pas noyer le moteur. J'aimais la sensation de puissance que donne la conduite du hors-bord, surtout le creusement central des eaux et leur exhaussement en éventail, l'intervention puissante qui crée le sillage et répercute ses effets jusqu'aux rives lointaines. Quand nous affrontions le courant, des panaches jaillissaient à chaque retombée de la proue, nous devenions un seul grand oiseau prenant sa course dans les régions basses du ciel, pour mourir et triompher de la mort. Oui... l'image fait un peu romantique ! C'est pourtant la sensation d'un absolu qui montait en moi, mêlée aux vibrations de tout le bateau et à son tintamarre 
qui chassait toute pensée; mêlée aux gifles du vent et des embruns. Pris dans un étui de bruit, d'air et d'eau, les yeux battus de souffles brutaux, je devenais la chose d'un désir plus grand que moi, coextensif à la vaste étendue d'eau qui s'arrondissait sous l'impulsion de mon élan.

L'hiver, la rivière devenait une grande plaine blanche ou parfois, après un redoux, une immense patinoire de plusieurs kilomètres de long. La côte derrière chez nous était assez haute pour que nous prenions plaisir à la descendre, soit en toboggan, soit en skis. Nous agrémentions la piste principale d'une butte de neige, au bas de la pente, qui transformait la glissade en tapecul.

Le ski de fond n'existait pas encore mais, avec nos skis alpins, nous faisions de longues promenades qui nous menaient dans les îles, en face de chez nous, ou plus loin, du côté de Saint-Eustache-sur-le-lac (aujourd'hui Deux-Montagnes) ou de Laval-ouest.

Selon l'épaisseur de la neige et le courage qui nous animait, nous tracions des patinoires plus ou moins vastes, que nous arrosions périodiquement grâce à un trou pratiqué dans la glace. Le déblayage et l'arrosage me plaisaient plus que les parties de hockey dont ils étaient le préambule nécessaire. Mon physique chétif me destinait par excellence au rôle d'arbitre, mais on me confiait parfois celui de gardien de but, pour le plus grand malheur de mon équipe. Quand les adultes s'affrontaient, les parties pouvaient être très animées, et les cris des joueurs et des spectateurs se répandaient jusque dans le village. Mon père, un jour, fit une chute et se frappa durement la tête sur la glace. Le coup le laissa étourdi pendant plusieurs minutes et lui enseigna cette prudence par laquelle les pères finissent par se comporter comme des pères et se retirer du hockey actif. La télévision, du reste, fit son entrée dans la maison, René Lecavalier remplaça Michel Normandin, Molson supplanta Dow et Terry Sawchuck battit Gerry McNeil. Nous ne pouvions plus jouer sans nous prendre pour Maurice Richard, Elmer Lach, Butch Bouchard ou Bernard Boum Boum Geoffrion, et nous commentions nos exploits avec la sagesse pataude et éraillée d'un Charlie Mayer ou de quelque autre pilier de la Ligue du Vieux Poêle. Parmi toutes ces scènes d'hiver, je me souviens surtout de quelques moments de bonheur absolu lorsque, étendu sur une congère, le regard perdu dans le bleu sans fond du ciel, que pas un nuage ne distrayait du merveilleux souci de sa limpidité, offert au soleil qui se faisait tout chaud, it me semblait sortir de I'hiver et du temps et entrer tout vif dans je ne sais quelle immortalité. J'éprouvais de semblables joies le soir, au spectacle de la lune et des grands arbres dont l'ombre s'imprimait nettement sur la neige, dans une immobilité pleine de mystère.

Mais Saint-Eustache, pour moi, ce n'est pas que la rivière, bien qu'elle occupe une place privilégiée dans mes souvenirs. Cela tient à mon côté farouche, qui me faisait fuir le contact des jeunes de mon âge, en dehors du cercle restreint de mes voisins. La rue où j'habitais, à cause de sa position au bord de l'eau, un peu en retrait par rapport au village, avait quelque chose de 
sélect, et la plupart de nos activités de loisir se rapportaient, égoïstement si je puis dire, à la rivière. Ce qui contribuait le plus à m'isoler, c'était une passion immodérée pour la lecture et même, dès l'âge de dix ans, pour l'écriture. Je passais beaucoup de temps dans la maison, où je trouvais du reste une protection contre les moustiques qui ont toujours raffolé de ma personne; contre l'herbe à puce qui me transformait, chaque été, en plaie suppurante de pied en cap, en momie rose de calamine; contre les coups de soleil, tout ce qui pique, cuit, érafle, égratigne. Cela dit, j'ai connu et aimé Saint-Eustache, les rues et les maisons plus que les gens, sans doute, et je voudrais évoquer quelques-uns des lieux qui m'ont marqué.

D'abord, l'école du Sacré-Cœur. J'y ai fait mes quatrième et cinquième années du primaire. Quand j'y suis arrivé, j'avais sept ans. Mes parents, qui me trouvaient des dispositions pour l'étude, m'avaient fait faire les deux premières années en une seule, en cours privés, à l'âge de cinq ans, et j'avais fait ma troisième année à six ans, dans un jardin d'enfants où les bonnes sceurs m'ont appris que cette vie n'est pas la vie, et que la terre est une vallée de larmes. Je découvrais donc l'école publique à sept ans et j'avais pour camarades des enfants âgés de deux ans de plus que moi. Les conditions n'étaient guère propices à mon intégration sociale, laquelle avait été fort mal engagée lors de mon année de bagne chez les religieuses. De ces années date le décalage dont j'ai longtemps souffert entre mon développement social et affectif et celui de mes facultés intellectuelles.

L'école était un cube de brique sévère, couleur de sang de bœuf depuis longtemps coagulé. De vieux érables l'ombrageaient et en atténuaient, pendant la belle saison, l'aspect rébarbatif. Les élèves entraient par une porte située à l'arrière du modeste édifice. Une odeur et des bruits de latrine nous accueillaient immédiatement et ne nous lâchaient plus, bien que la densité s'en perdît quelque peu à chaque étage.

Quand la cloche sonnait, avant l'ouverture des portes, le matin ou au début de l'après-midi, il fallait nous mettre en rangs, deux par deux, toujours dans le même ordre, les plus petits en avant et les plus grands en arrière. Nous formions des colonnes savamment graduées, pour des fins dont j'ignore si elles ètaient esthétiques ou militaires. L'esprit militaire était, de toute façon, très présent à l'école. En 1949, la guerre était à peine chose du passé. Tous les jours il y avait la cérémonie du salut au drapeau. Les écoliers étaient enrégimentés de force dans la compagnie dite des Cadets de SaintEustache. J'entends encore nos officiers, adolescents butors qui se prenaient pour Rommel, crier avec une absolue conviction: "ADETS DE SAINT'USTA'HE, EN A'ANT..." ARCHE!" La marche était stimulée par le contrechant suivant: "And a left, and a left, and a left, right, left!" On entendait parfois la traduction: "And a gauche, and a gauche, and a gauche, drette, gauche!" Ces anapestes, tantôt couronnés de spondées, réveillaient dans nos âmes des fiertés homériques. Nous portions, dans les parades, des pantalons noirs à galon rouge, une veste rouge et un "caluron" (est-ce la déformation du mot galurin?) tout rond, qui achevait de nous donner une allure assez semblable à celle du Fifre, de Manet. 
Les plus grands, élèves de sixième ou de septième année, formaient la fanfare. Il y avait les tambours, les clairons, qui sont la condition sine qua non d'une fanfare, une grosse caisse et un tuba qui donnaient le frisson sacré. Le verbe québécois intransitif fesser (exemple: "Maudit que ça fesse! Ça fesse donc dur!") correspond exactement aux percussions dont notre brave jeunesse assommait ses tambours, cependant que les cuivres cherchaient, avec des cris déchirants, la ligne mélodique de " $A$ la claire fontaine", ramenée à trois notes praticables.

Nous avions fière allure, certes, mais jamais autant que les adultes quand ils se mettaient de la partie. Je me rappelle une gloire locale, major de l'armée canadienne, avec son béret, sa moustache et sa culotte courte. Le principal de notre école revêtait un costume semblable, dans les grandes circonstances, et j'en étais tout gêné pour lui, moi qui pouvais enfin porter le pantalon. Mais les plus flamboyants, aux processions de la Fête-Dieu, étaient les officiers des chevaliers de Colomb. Ils portaient le haut de forme et une cape noire doublée de satin rouge, dont les bords étaient rabattus, ce qui leur donnait l'air d'Arsène Lupin; au poing, ils avaient leur épée toute droite, comme un parapluie sans chapeau.

Je reviens à l'école, qui était la caserne à partir de laquelle rayonnait notre vertu militaire. Elle était aussi - autre temps, autres mœurs - un lieu d'apprentissage. On nous enseignait le pire et le meilleur, la bienséance et la grammaire, le calcul et l'histoire sainte, le petit catéchisme et l'histoire du Canada.

Mon maître, en quatrième année, était un jeune clerc de Saint-Viateur, frais émoulu du juvénat. II nous racontait souvent le sacrifice de sa vie amoureuse, l'adieu à sa petite amie qui ignorait jusque-là sa vocation, et l'espoir qu'il avait d'aller au ciel tout droit, en reconnaissance de ses mérites. II avait beaucoup d'autorité, lançait le tampon à effacer sur ceux qui somnolaient ou dont l'esprit battait la campagne, donnait des coups de sa courroie de caoutchouc spéciale, la "strappe", aux enfants dissipés ou à ceux qui avaient mal retenu la leçon.

On l'aurait cru diplômé de la faculté des Sciences de l'éducation, un quart de siècle avant sa fondation, tant il manifestait d'astuce pédagogique. Ainsi un jour, avant la prière qui devait commencer le cours, il se promena dans les rangs et dit, à cinq ou six de mes camarades: «Debout, en avant! » Puis il demanda: “Savez-vous pourquoi j'ai fait mettre vos petits camarades en avant de la classe? "Nous risquâmes quelques hypothèses, aussitôt refusées. "Si j'ai fait mettre vos petits camarades en avant de la classe, finit-il par expliquer, c'est qu'ils ont les oreilles sales! crottées! Untel, par exemple, on dirait de vrais nids à souris. Ta mère ne te lave donc pas?"Nous comprîmes que débutait une leçon d'hygiène.

L'année suivante, les clercs avaient plié bagage et une administration laïque leur avait succédé. Mon maître, en cinquième, était un vieil original, qui avait ses protégés et qui passait beaucoup de temps à nous conter des fariboles, sur la lune notamment où vivaient des êtres verts à ventre bleu, et 
où les autobus étaient des sauterelles de trente pieds de long. Nous portions sur lui le même jugement que Platon sur les poètes: nous le trouvions menteur. Ses mensonges, pourtant, nous enchantaient, et nous en redemandions.

Instituteur par métier, et non par vocation, il n'avait guère d'estime pour les enfants, sauf ceux dont les parents l'honoraient de quelque amitié. II se moquait bien de nous, dans le fond, et c'est sans doute pour le plaisir de me voir blêmir que, lors d'un pique-nique, il me fit fumer huit cigarettes, les plus fortes sur le marché. J'avais neuf ans, je fus affreusement malade. « Si ça a du bon sens!" commentèrent mes parents, déjà portés à s'interroger sur les avantages du secteur public.

Un autre lieu qui compta pour moi fut l'église, l'historique église de Saint-Eustache, dont la façade conserve jalousement la trace des boulets anglais de la Rébellion. Sans doute ces stigmates ont-ils pour raison d'être de convaincre les incrédules : l'apôtre Thomas n'avait pu croire à la résurrection du Christ sans vérifier, du bout des doigts, la présence des saints trous. Ainsi était-il difficile, pour ceux qui connaissaient les combats de Saint-Eustache par le récit des frères des Écoles chrétiennes et les gravures qui l'illustraient, de faire le lien avec la réalité. L'aspect de l'église, son emplacement, la disposition des rues, rien de tout cela ne concordait. Seuls les trous attestaient le passage de Colborne et des habits rouges.

Nous n'allions pas régulièrement à l'église de Saint-Eustache, celle de la paroisse Saint-Agapit, à Saint-Eustache-sur-le-lac, étant aussi proche de chez nous et nous étant plus sympathique. Pendant l'année scolaire, je faisais cependant partie de la manécanterie de mon village. J'ai donc bien connu l'église, mais surtout son jubé. A cause de mon état de chanteur, je n'ai jamais appris à servir la messe, ce qui constitue l'une des trois lacunes de mon existence, les deux autres étant mon inaptitude à parler anglais et à conduire.

L'église de Saint-Eustache m'impressionnait beaucoup par ses dimensions et par une sorte d'austérité. Les boiseries sombres ont toujours produit sur moi un effet déplorable, elles me plongent dans le spleen le plus total. Le chœur était ceinturé de grands tableaux qui rappelaient la vie de saint Eustache. L'un d'eux le montre en arrêt devant un cerf qui porte, entre ses bois, une croix lumineuse. Un autre peint son martyre: il exulte, insoucieux des flammes, dans une chaudière de bronze. Sans doute son destin annonçait-il, en moins laïc, celui de Chénier, que le feu allait chasser de l'église assiégée.

Je n'ai pas pénétré dans l'église depuis une bonne vingtaine d'années; j'ignore si le vent de modernisation qui a soufflé sur les édifices consacrés au culte l'a touchée et, si oui, dans quelle mesure. Je me souviens surtout de l'œil de cyclope encadré d'un triangle qui, du plafond, à l'intersection de la nef et du sanctuaire, plongeait son regard inquisiteur dans nos consciences. Cet œil sans corps, tout entier livré au plaisir de voir, me fascinait. Dès l'âge de six ans, j'ai été sujet, dans les églises, à des étourdissements qui allaient jusqu'à la syncope. Le fameux œil y était pour quelque chose. 
Le souvenir le plus vivace qui me soit resté de ma fréquentation de l'église est celui d'une humiliation. J'avais neuf ans. J'aurais dû recevoir le sacrement de confirmation à sept ans, mais une épidémie de rougeole avait mis fin à mon calvaire au jardin d'enfants de Rigaud, où j'étais pensionnaire, et je n'avais pas été confirmé. Je ne le fus pas non plus l'année suivante car, si je me souviens bien, la cérémonie n'avait lieu alors que tous les deux ans. Quoiqu'il en soit, j'étais en cinquième année et, seul de ma classe, je devais suivre les exercices préparatoires avec les élèves de troisième et de quatrième, qui du reste n'étaient guère plus jeunes que moi. Tout alla bien, et le grand jour arriva. J'avais revêtu mon plus beau costume, et j'étais dans des dispositions fort pieuses. Celui qui devait nous administrer le sacrement était le nouvel archevêque de Montréal. Entre enfants, nous examinions des hypothèses plus ou moins rassurantes concernant la violence du soufflet qu'il nous donnerait. C'était affaire de sadisme personnel, mais aussi d'efficacité spirituelle: un soufflet bien appliqué devait avoir plus de valeur qu'un attouchement furtif. "Moi, s'il me fait mal, je lui donne un coup de pied!" déclarait un fanfaron, dans la plus pure tradition des Patriotes.

A peine entré dans l'église, ce jour-là, je restai saisi par la fraîcheur du lieu et me mis à éternuer. Je portai aussitôt la main à ma poche pour y prendre mon mouchoir. Ma poche droite ne contenait que mon chapelet. Je visitai ma poche gauche, les poches arrière, mon veston tout entier: pas de mouchoir et rien, absolument rien qui pût en tenir lieu. Gargantua s'était fait un torchecul avec le cache-nez de velours d'une demoiselle, avec un cachecol, avec un bonnet de page, avec de la sauge, du fenouil, de l'aneth, de la marjolaine, les draps, les couvertures, les rideaux et cent autres choses. Je n'étais pas Gargantua, et mon imagination resta en panne. Impossible de me lever et d'aller trouver mes parents, placés dans une section de la nef éloignée de celle où nous étions. La cérémonie était commencée et allait se dérouler avec une rigueur mécanique. Malheur à qui en perturberait le bon fonctionnement. On nous avait recommandé la piété la plus stricte. J'étais pris dans l'engrenage, rien ne me ferait échapper à mon destin. Heureusement, mon voisin de banc était mon voisin dans la vie, et j'osai m'adresser à lui : "Tu n'aurais pas un kleenex? " Nous ne nous aimions guère, et il me fit répéter trois fois ma demande. II faut dire que je chuchotais le plus bas qu'il m'était possible, pour ne pas attirer l'attention du terrible vicaire qui était responsable des confirmations et qui nous surveillait par derrière. Finalement, mon copain répondit qu'il n'avait pas de kleenex, mais seulement un beau mouchoir brodé. Je lui demandai s'il pouvait me le prêter... rien qu'un peu. II me dit que sa maman ne voudrait pas. Sa mère, en effet, m'inspirait de la terreur, elle était du genre acariâtre, et je n'insistai pas. Pour dissimuler l'état de mon visage, je joignis les mains et les approchai de mon nez. La cérémonie avançait et il faudrait bientôt se mettre en rang pour défiler devant l'archevêque. Je le voyais, de loin, dans son accoutrement excentrique, et priais Dieu de faire un miracle. Mais Dieu se bidonnait, et j'avais beau essayer de me fasciner sur le triangle du plafond et le gros cil bleu, je n'éprouvai pas le moindre vertige. Un évanouissement, en effet, m'eût tiré d'affaire. Ce secours m'était refusé. J'étais vaillant comme jamais, mon cceur pompait le sang avec 
pétulance dans mes veines, je pissais presque le sang par les oreilles, les larmes par les yeux et, en tout cas, la morve par le nez.

La file se constitua. La tête bizarrement inclinée sur mes mains jointes, j'avançai comme au supplice, avec une morne résignation. J'aboutis ainsi jusqu'au pied de l'escalier en haut duquel trônait l'homme de Dieu. Le vicaire terrible, qui l'assistait, m'aperçut alors et, d'un geste impérieux, me signifia l'ordre de me moucher. Je répondis, par un geste timide et désespéré, que j'étais dans l'incapacité de le faire. II fut pris au dépourvu, leva les yeux au ciel et m'oublia. Un des enfants, devant moi, impressionné par la tape qu'il avait reçue, sanglotait dans son mouchoir, l'heureux imbécile! J'arrivai enfin devant le prélat. II avait l'air sévère et fatigué. II prononça des formules latines, me regarda, hésita un moment puis, paf! m'asséna une claque à étourdir un chrétien. J'en fus presque mouché du coup, les mucosités volèrent et je me retrouvai confirmé, confirmé dans la honte et le ridicule. Jamais je n'avais subi pareille humiliation. En même temps que le caractère sacré s'écrivait dans mon âme, le temps d'une gifle, j'y sentais poindre le ferment de rancour qui m'anime encore parfois contre l'Église, ses pompes et ses œuvres. Quant à l'archevêque, il dut être marqué lui aussi: depuis la claque qu'il me donna, il prit du goût pour les lépreux.

Comme on le voit, l'école et l'église ne sont pas liées, dans ma mémoire, qu'à d'agréables souvenirs, loin de là. C'est que j'avais peu de goût pour l'expérience grégaire, et que j'étais maladroit à intérioriser l'ordre social. Mal doué pour le jeu, surtout les jeux d'équipe qui demandaient des habiletés physiques que je n'avais pas, je restais à l'écart de mes camarades, dont les mcurs brutales m'effrayaient. Quant aux adultes, ils m'inspiraient soit de la crainte, soit du mépris. J'avais ri aux larmes d'une dame corpulente, venue rendre visite à ma mère et qui s'était écriée, avant même de saluer : «Hé! ma chèrrrre enfant, j'ai mis mon corset parce que c'est effrrrrayant comme ça fait des grosses fesses quand on n'en met pas! "J'aurais tort, bien entendu, de généraliser et de voir, en cette bonne dame, un symbole de la faune pensante de Saint-Eustache. II n'empêche que, la lecture de Rimbaud aidant, je n'avais pas d'affection démesurée pour mon Charleville natal. Dès l'âge de neuf ans, je commençai à voyager, matin et soir, entre Saint-Eustache et le collège, sis en plein centre de Montréal. II fallait me rendre à la gare de Deux-Montagnes, à un mille de chez moi, et faire cinquante minutes de train. Une fois descendu à la Gare centrale, je marchais pendant dix autres minutes et j'étais enfin rendu. Ces efforts ne me coûtaient guère: Montréal est un soleil, SaintEustache est son satellite. Il est une ville-dortoir, comme on dit si justement. C'est la ville où l'on dort, de gré ou de force, parce qu'il n'y a rien d'autre à y faire. Je vivais, étudiais, souffrais et aimais à Montréal. De retour chez moi, je cherchais le refuge de ma chambre, où je passais pour étudier beaucoup. Davantage je lisais, et davantage encore je rimais, rêvant d'égaler les plus grands. En fait c'est cela, je rêvais, et comme tous les rêves un jour prennent fin, je me réveillai critique littéraire.

Si j'avais mieux aimé mon pays, ma petite patrie, j'aurais pu devenir romancier et écrire les Mystères de Saint-Eustache; ou changer les trous de 
mon église, et je serais un autre Nérée Beauchemin. Mais non! je riais, sottement, des poèmes de mon vieux compatriote, épicier nostalgique, dans la gazette locale. II est bien vengé aujourd'hui. " $O$ temps, suspends ton vol! heures propices / Laissez-nous savourer les rapides délices / Du plus beau temps de nos jours!" Moi seul, critique, suis en mesure d'évaluer les écorchures faites au texte de Lamartine, l'horreur du pléonasme de la fin; et lui, dans sa tombe, il rit, de toutes ses dents rapportées.

L'école, l'église, le village, je les aime parce qu'ils sont mon passé, mais quand ce passé était mon présent, je les trouvais certes durs à vivre. Aujourd'hui, je peux les revoir avec plaisir, du moins les deux derniers car l'école, la pauvre, a été rasée, il n'en reste pas brique sur brique, et le lieu où j'ai ânonné les cinq cent huit réponses du petit catéchisme, rendu à la paix de ses beaux arbres, est maintenant un jardin public. Quelque Roquentin, un de ces jours, ira y méditer sur l'existence, devant une racine bien de chez nous. Je n'ai pas de chance avec les écoles: le collège Sainte-Marie, où j'ai fait mes études classiques, a basculé lui aussi dans le néant. Heureusement, l'Université de Montréal ne semble pas devoir m'inspirer de crainte, du moins quant à son avenir matériel.

J'ai commencé ce petit voyage au pays de mon enfance en évoquant la grande et belle rivière auprès de laquelle j'ai grandi et à qui je dois mes joies les plus nombreuses. Je le terminerai par une promenade nonchalante, dans mon petit bateau à rames, sur une autre rivière qui, elle, est ignorée des cartes géographiques, et qui tranche Saint-Eustache en deux parties, comme la Seine tranche Paris. C'est la rivière du Chêne, dont j'écris le nom en deux mots, faute de savoir à quoi m'en tenir et parce que cela me plaît mieux ainsi. Petit affluent de la rivière des Mille-Iles, la rivière du Chêne n'a pas le charme de la Seine, pas plus que Saint-Eustache ne rivalise, sous quelque rapport que ce soit, avec Paris (France). Mais il est un point commun aux deux cours d'eau. La rivière eustachoise, en effet, reçoit les eaux usées de toute la ville, ce qui en affecte grandement la limpidité. Aux jours les plus chauds du mois d'août, il n'est pas recommandé de s'y aventurer sans masque à gaz ou quelque protection équivalente. Là frétillent la barbote et le crapet en abondance. Les pêcheurs qui ne répugnent pas à ces nourritures le savent et font des prises dignes de Saint-Pierre.

L'embouchure du modeste cours d'eau n'est guère éloignée de la maison de mon enfance. Entre les deux se trouvent quelques propriétés et ce qui reste d'un petit bois où j'ai beaucoup joué à l'Indien, dans mon jeune âge. Puis se trouve l'ancien emplacement d'une industrie de produits marinés et de confitures. Avant l'incendie qui a entraîné sa fermeture, il y avait d'immenses cuves de bois où trempaient, dans une saumure glauque, des cornichons de toutes tailles. Ils patientaient là pendant des mois, peut-être des années, comme les âmes du purgatoire, attendant leur promotion. Des passerelles donnaient accès aux cuves, laissées le plus souvent à découvert. Tout ce qui tombait du ciel allait tenir compagnie aux cucurbitacées - la pluie, la neige, les fientes d'oiseau, tout un peuple d'insectes amateurs de saumure, sans compter ce que les petits anges du quartier, j'étais du nombre, 
y laissaient choir. La surveillance était inexistante, mais je n'ai jamais entendu dire qu'on eût retiré de cadavre humain de ces solutions croupies.

Juste à côté, une laiterie déversait naguère ses eaux blanches dans la rivière, à l'embouchure même du petit cours d'eau qui lui était redevable, en bonne partie, de sa couleur trouble et de son odeur. Ici commence vraiment notre promenade. A l'arrière du canot, là oủ la petite et la grande rivière se rejoignent, se dresse l'ile du Curé, petit morceau de terre couvert de forêt vierge et d'herbe à puce. J'ignore l'origine de son nom, qui procède peut-être tout bonnement d'une logique métonymique. En effet, la rive droite de la rivière du Chêne, à quelques mètres de l'lle du Curé, fait partie du vaste terrain où s'élèvent l'église et le presbytère. Ce dernier est une confortable construction de pierre grise, entourée de vieux arbres magnifiques. Sa vue rachète celle des eaux épaisses, où flottent un tas d'objets non identifiables (ofnis).

Sur la rive gauche, la laiterie était un mauvais moment à passer. Voici maintenant une propriété superbe, autrefois celle de Chénier puis, plus récemment, de Paul Sauvé qui fut, pendant une centaine de jours, premier ministre du Québec. Elle appartient maintenant au député péquiste. Une vaste maison de bois, blanche, domine la pelouse ombragée qui descend jusqu'à un quai de pierre. Tout cela, avec le presbytère sur l'autre rive, fait très aristocratique.

Mais voici maintenant le "petit pont", qui doit sa dénomination à une comparaison implicite avec le pont Sauvé qui enjambe, plus loin, la rivière des Mille-Iles. Quand je passe dessous, l'eau qui dégoutte des rames acquiert une sonorité métallique. Si je criais au meurtre, l'écho me tuerait tant la résonance est amplifiée par les énormes poutres d'acier! Une fraîcheur lourde de sous-bois tombe sur mes épaules. Le passage des voitures produit un grondement de catastrophe. Mais voilà assez d'hyperboles, je sors de la pénombre, lance un coup d'œil inquiet au-dessus de moi où des galopins pourraient bien avoir l'idée de me jeter des caillous. Non, tout va bien et je débouche sur une rivière bien différente, étroite, serpentine; à droite s'entassent les horreurs commerciales de la rue Saint-Eustache, dont on ne voit que les derrières. Un totem grotesque signale un bar soi-disant indien, et qui le dit en anglais. De beaux saules pleureurs masquent un peu la laideur des lieux. Sur l'autre rive, le cimetière vient expirer, ce qui est bien dans sa nature. L'eau est de moins en moins profonde et il faut prendre garde de ne pas s'envaser. On a l'impression de toucher bientôt au terme du cours navigable. Cependant, voici apparaître à droite une magnifique maison de style canadien, toute blanche, au toit vert. Un potager soigneusement entretenu dévale la côte, et d'immenses maisons d'oiseaux abritent les hirondelles bicolores et les hirondelles pourprées. La rivière s'élargit alors de nouveau et prend même l'aspect d'un petit lac, bordé à gauche d'une végétation touffue et sans rien qui la dépare. Mais si le regard longe la rive droite, il découvre soudain la surprise des surprises - et le but de notre expédition. C'est la Dame! la merveilleuse Dame à Légaré! Celle où les puristes ne verront qu'un modeste barrage, mais où les passionnés, les 
amoureux, les poètes et les enfants voient la Dame, la Dame qui chante et qui égare, la Dame à Légaré.

Et Légaré, c'est le moulin plus que centenaire, dont la maçonnerie en pierres des champs s'élève, depuis le lit de la rivière, jusqu'à une hauteur de quinze mètres! Un pur joyau de l'architecture québécoise d'autrefois, vrai poème de pierre enté à la rivière, par la grâce de la digue qui laisse l'eau s'épandre en nappes claires et musicales. Que murmure-t-elle depuis tant d'ans, comme une source chuchoteuse, la Dame à son moulin amant, que ditelle de si émouvant, que j'ai l'âme qui tremble en l'écoutant?

Je n'avironne plus. Je m'échoue peut-être, peu importe. Il y a le grand calme des lieux. Il y a la lumière, le verdure et la grande fraîcheur de tout.

Au cœur de la ville, il y a cette sonate de l'eau et de la meule.

On y moud le blé, l'avoine et le sarrazin.

On y change les pierres en pain.

Au cœur de la ville, il y a cette aurore du passé resté enfant, comme une chanson très simple qui brille au fond des temps.

Je reste là, immobile, un bon moment.

Puis je reprends les avirons. Je redémarre doucement. La visite est terminée. Adieu, ma douce, adieu. C'est moi, ton égaré'.

André BROCHU

1. Ce texte a èté présenté à l'émission «Un écrivain et son pays" sur les ondes de Radio-Canada FM, le 11 août 1979. 